Katarzyna Walkowska ${ }^{1}$

Uniwersytet im. Adama Mickiewicza

\title{
Interkulturelles Lernen und Lehren im Fremdsprachenunterricht - warum, wozu und wie?
}

\begin{abstract}
Summary
The aim of this article is to discuss the problem of intercultural competence and communication. The author wants to draw attention to the phenomenon of the concept of culture and its role in the process of developing intercultural communication and intercultural competence.
\end{abstract}

Keywords: foreign language teaching, intercultural competence, intercultural communication

\section{Einleitung}

Der Anfang des dritten Jahrhunderts hat das humanistische Milieu in dem Zustand gleichzeitig der Unsicherheit und der Hoffnung angetroffen (vgl. Grzybowski 2011: 22). Die seit den 1960er Jahren permanenten Migrationswellen nach Mittelund Osteuropa, der heute viel zitierte und besprochene Globalisierungsprozess, die Entwicklung von Tourismus verlangen neue Wege, Möglichkeiten und Mittel, die daraus folgende Annäherung von Menschen und ihren Kulturen wirksam und ohne Konflikte zu gestalten. Seit Beginn der 90er Jahre konnte man in den Industrienationen der Welt wieder ein verstärkter Zuwanderungsstrom beobachten. Sprachliche Vielfalt und Kommunikation und Sprachbegegnungen beeinflussen zunehmend die schulische Realität. Kein Wunder, dass sie zu relevanten pädagogischen und bildungspolitischen Anforderungen geworden sind (Schmölzer-Eibinger 2007: 156).

Beim Überschreiten der Grenzen, beim steigenden Tourismusverkehr und demzufolge beim Kontakt mit Mitgliedern anderer Kulturen spielt die Sprache immer wichtigere Rolle ab. Sie sollte nicht nur als Informationsübermittlungsinstrument wahrgenommen werden. Die Sprache wird in der globalisierten Welt eines des wichtigsten Kommunikationsinstruments unserer Zeit. Ihre Bedeutung wird aber heute oft unterschätzt.

1 https://orcid.org/0000-0003-3596-5366. 
Während bis Ende der 1970er Jahre die traditionelle Landeskunde Kernbereich des schulischen Fremdsprachenunterrichts war, musste man auf die wachsende Entwicklung in den Bezugswissenschaften und der Fremdsprachendidaktik, sowie auf die gesellschaftliche Entwicklung und ihre Anforderungen durch ein neues Konzept reagieren. Die Antwort auf daraus resultierenden Fragen und Problemen versuchte die in den Erziehungswissenschaften entstandene Ausländerpädagogik $\mathrm{zu}$ finden, die auch $\mathrm{zu}$ Veränderungen in dem Fremdsprachenunterricht beitrug (vgl. Burwitz-Melzer 2003: 38f.). Das Hauptkonzept der Fachdiskussion um die Integration von Schülerinnen und Schülern, die unterschiedliche Herkunft und Muttersprache haben, ist seit Beginn der 80er Jahre die Konzeption des interkulturellen Lernens. Interkulturelles Lernen wird in diesem Sinne als Erziehung zu kultureller Offenheit und zur Überwindung von Ethnozentrismus und Rassismus (Schmölzer-Eibinger 2007: 156f.). Dem Konzept gemäß sollte die Schule die Idee der Toleranz fördern. Sie sollte die Lernenden aufklären, dass jede Kultur ihre ganz spezifischen Merkmale besitzt und durch eigene Arten des Erlebens und der Verhaltensmuster geprägt wird (ebd.).

Es unterliegt keinem Zweifel, dass all diese Prozesse ohne Teilnahme von Kommunikation nicht möglich wären. Deswegen wird der Begriff der interkulturellen Kommunikation in der Fremdsprachendidaktik allgemein verwendet. Viele stellen sich aber die Frage, was diese interkulturelle Kommunikation eigentlich ist. Widawska (2006: 22) betont, dass fremdsprachige Kommunikation, die immer kulturelle Elemente enthält, mit der nationalen und fremden Kultur verbunden ist. Aus diesem Grund ist es relevant, den Begriff „Kultur“ genauer zu besprechen.

\section{Kultur - Begriffsannäherung}

Für den Begriff „Kultur“ ist eine terminologische Vielfalt (AdamczakKrysztofowicz 2003: 19) charakteristisch. Man spricht von dem traditionellen (Kretzenbacher 1992), anders gesagt, einem engen Kulturbegriff (Seelye 1976) oder auch von Kultur im engeren Sinne (Benecke 1995). Im Gegensatz dazu funktioniert die naturwissenschaftliche und anthropologische Bedeutung dieses Terminus (ebd.), die zur Entstehung des erweiterten Kulturbegriffs (Seelye 1976, Szczodrowski 1997) führt. Zum Schluss benutzt man die Definition der „fremdsprachigen Kultur“ (Pfeiffer 2001, Kaikkonen 1997), die eng mit dem interkulturellen Ansatz im fremdsprachlichen Unterricht verbunden wird. 
Das Kulturphänomen besteht darin, dass sie viele Interpretationen zulässt. In den Printmedien wird sie aus der Perspektive der Kunst, künstlerischer Leistungen, Errungenschaften einer Nation usw. wahrgenommen. In den elektronischen Medien wird Kultur mit Sport und Unterhaltung assoziiert. In der Touristikbranche bezieht sich Kultur auf das kulturelle Angebot und wird vielfach als ein Element der Freizeitgestaltung verstanden (ebd.). Der Kulturbegriff gewinnt auch in der Fremdsprachendidaktik an Bedeutung. Aus diesem Grund ist es notwendig, den Begriff „Kultur“ zu definieren, um die Gefahr von Missverständnissen zu reduzieren.

Man sollte mit der Erklärung der Etymologie des Wortes „Kultur“ beginnen. Der Kulturbegriff - cultura, cultus - kommt aus dem Latein und wurde vom Wort colo, colere, colui, cultam abgeleitet. Im klassischen Latein war cultura ein landwirtschaftliches Fachwort und bedeutete „Landbau“, „Pflege (des Körpers und Geistes)“ (vgl. DUDEN 2007: 1028). Ein neues Kulturkonzept ist Cicero zuzuschreiben, der seine metaphorische Definition der Philosophie als „cultura animi“ bezeichnete. Diese metaphorische Kulturauffassung bleibt in der europäischen Geistesgeschichte bis ins 19. Jahrhundert lebendig (vgl. Mihułka 2008: 14).

Es gibt viele Kulturdefinitionen, die oft voneinander abweichend und sogar widersprüchlich sind. Aus diesem Grund sind die Worte des deutschen Philosophen Gottfried Herder sehr bedeutend, der schon im 18. Jahrhundert im Vorwort zu den „Ideen zur Philosophie der Geschichte der Menschheit“ (17841791) geschrieben hat, dass es nichts so Unbestimmtes gibt, wie Kultur.

Für das interkulturelle Lernen ist bei der Definition die Berücksichtigung der unterschiedlichen und voneinander verknüpften Elemente von Kultur (wie z.B. Sprache, Politik, Geschichte, Literatur, Kunst etc.) sehr relevant (vgl. Keller 1996: 228, in Jańska 2006: 20). Wenn man eine fremde Sprache lernt, lernt man auch die Kultur des Zielsprachenlandes kennen (Mihułka 2010: 23), denn „Fremdsprachenlernen heißt: Zugang zu einer anderen Kultur suchen“ (Krumm 1994: 28). Im Fremdsprachenunterricht kommt es zum "Zusammenstoßen" der eigenen mit der fremden Kultur, zur Begegnung der unterschiedlichen Normen, Regeln und Tradition. Die neue - andere Kultur wird mit der eigenen konfrontiert und durch das Prisma der eigenen Kultur wahrgenommen (Mihułka 2010: 24).

Im Fremdsprachenunterricht vermittelt man die Aspekte der eigenen und der fremden Kultur, das unbedingte Wissen, was den Lernenden ermöglicht, alle Unterschiede und Zusammenhänge zwischen den beiden Kulturen zu finden, sie angemessen $\mathrm{zu}$ interpretieren und $\mathrm{zu}$ verstehen. Ohne dieses Wissens 
ist es unmöglich, sich entsprechend der Situation zu verhalten, auf die bestehende Situation adäquat zu reagieren und zu agieren (ebd.).

Schon Göhring (1980) hat eine erfolgreiche Definition vom Kulturwissen geschaffen. Die von ihm umgefasste Definition enthält das Wissen über eigene und fremde Kultur, die in einem Zusammenhang gemeinsam stehen. Des Weiteren führt er aus, dass die Kultur alles ist, was ein Individuum wissen muss, um im Stande zu sein, zu beurteilen, ob und in welchem Maße das Verhalten der Einheimischen unseren Erwartungen entspricht und wo sich ihre Verhaltensweise von unseren Erwartungen unterscheidet.

Kultur wird manchmal als eine Gruppe selbst, die durch eine gemeinsame Lebensweise markiert ist, nicht als eine Lebensweise einer Gruppe, definiert. Brislin (1981: 3) neigt zu dieser Definition, für den eine Kultur mit einer identifizierbaren Gruppe als gleich angesehen ist, in der gemeinsame Normen funktionieren, gemeinsame Werte geachtet und gemeinsame Erfahrungen gesammelt werden.

Kultur wird auch oft mit Kommunikation assoziiert. Der amerikanische Anthropologe Edward T. Hall hat in 1959 die kürzeste Definition der Kommunikation und Kultur formuliert, indem er zugleich betonte, dass Kultur Kommunikation und Kommunikation Kultur sei. Das bedeutet, dass Kultur, in der wir leben, den Kommunikationsstil bestimmt, und umgekehrt der Kommunikationsstil modifiziert unsere Kultur (vgl. Szopski 2005: 5). Szopski betont weiter, dass wir unsere Kultur im Sozialisationsprozess lernen, deshalb kommunizieren die Mitglieder verschiedener Kulturen anders (ebd.).

Jede konkrete Gesellschaft, jedes Individuum hat seinen eigenen Platz in Zeit und Raum. In dieser Zeit-Raum-Dimension verarbeitet der Mensch die Innen- und Außenwelt. Als Folge von vielen Erfahrungen, die er heutzutage macht, ändert er nicht nur seine soziale und kulturelle Lage, sondern er verarbeitet auch seine Welt (vgl. Leoński 2005: 12). Der Begriff "Kultur" ist in der Gesellschaft allgemein bekannt und wird sehr oft verwendet. Er ist auch für Methodiker und Wissenschaftler, die sich mit dem Thema ,interkulturelle Kompetenz" befassen, ein Schlüsselbegriff. Die Erklärung und Definition dieses Begriffs ist eine Basis für weitere Überlegungen. Es unterliegt keinem Zweifel, dass der Begriff „Kultur“ gegenwärtig und in verschiedenen Kontextbezügen verwendet wird. Dies betrifft nicht nur den wissenschaftlichen Dialog in den Fachkreisen, sondern auch die alltägliche Kommunikation. 


\section{Kultur und Sprache - Verhältnisse zwischen den beiden Begriffen}

Viele Wissenschaftler behaupten, dass die Sprache ein Kulturträger ist, oder dass sie den Menschen einen Zugang zur Kultur, die sie kennen, ermöglicht. Man benutzt auch eine Metapher, nach der die Sprache ein „Schlüssel“ zu Kultur ist (vgl. Banach 2003: 3). Die gegenseitigen Zusammenhänge zwischen Sprache und Kultur haben Philosophen, Sprachwissenschaftler und Soziologen reflektiert.

Einer der ersten Forscher, der Aufmerksamkeit auf die Einheit der Sprache und Kultur gelenkt hat, war Wilhelm von Humboldt - der deutsche Sprachwissenschaftler, der um die Wende von 18. zum 19. Jahrhundert gelebt hat. Nach Humboldt gibt es eine gegenseitige Korrelation zwischen Sprache und Kultur, weil jede Kultur mit der Nationalität verbunden ist. Einerseits bildet die Nationalität die Sprache und andererseits ist es umgekehrt - die Sprache drückt den Geist der bestimmten Nationalität aus (vgl. Koniewicz 1997: 306f.):

Język jest rezultatem ducha narodu, emanacją jego najbardziej istotnych cech. Nie oznacza to wszakże, iżby nie wywierał on zwrotnego wpływu na życie narodów - duch narodu jest wprawdzie przyczyną sprawczą języka, tym niemniej sam podlega oddziaływaniom ze strony wywołanego przez siebie skutku. Język stanowi, jak powiada Humboldt, pierwszy i konieczny szczebel, z którego naród zaczyna piąć się $\mathrm{w}$ górę ku wyższym etapom swego kulturowego rozwoju. To obopólne powiązanie rozwoju języka oraz innych zakresów kultury są nierozerwalne $\mathrm{i}$ towarzyszą sobie od początku historii danego narodu (Andrzejewski 2005: 40).

Daraus folgt, dass die Sprache ein integraler Teil der Kultur ist. Sie ist sowohl ein Kulturträger, als auch der empfindlichste Messer ihrer Verwandlungen. Sie ist ein Bindemittel der kulturellen Gesellschaften und das Fremdsprachenlernen ist das "Öffnen des Fensters" zu der fremden Kultur (vgl. Aleksandrowicz-Pędlich 2005: 13).

Eine sehr wichtige Rolle bei der Erforschung von Zusammenhängen zwischen Sprache und Kultur hat Malinowski gespielt. Er behauptet, dass die Sprache in der Kultur einer Nationalität „verankert“ sei, und den Glauben und die Gefühle einer Gesellschaft ausdrücke:

(...) language is essentially rooted in the reality of the culture, the tribal life and customs of the people, and ... it cannot be explained without constant reference to these broader concepts of verbal utterance (Malinowski 1923: 305). 
In der Fachliteratur wird zwischen sprachlichem Universalismus und extremem Relativismus differenziert. Nach Ansicht von Universalisten wird die Sprache als ein autonomer Bereich wahrgenommen. Diese Theorie besteht darin, dass dieselbe Information in verschiedenen Sprachen formuliert werden kann. Die Befürworter des sprachlichen Relativismus sind Anhänger der sog. Sapir-Whorf-Hypothese vom Anfang des 20. Jahrhunderts, die davon ausgeht, dass das Denken durch die Sprache bestimmt wird.

Pawłowska (2009: 58) betont, dass die Sprache einer Gesellschaft ohne Bezug auf die Kultur, mit der sie eine untrennbare Ganzheit bildet, nicht betrachtet werden kann. Wie Odelski (2005: 22f.) bemerkt, stellt die Sprache als ein Kommunikationsmedium der bestimmten Intentionen und Inhalte dar. Sie ist eine „Vorpost“ unserer Meinungen, manifestiert unsere Emotionen, steckt das Gebiet ab, außerhalb dessen kann die Welt existieren (und oft existiert), die wenn nicht neutral, dann so konstruiert wird, dass sie weit von menschlichen Meinungen, Glauben und Träumen ist. Das ist die Welt gegenüber deren, sich unser alltägliches Streben nach dem Ziel mit dem Wort wehrt (ebd.).

Kramsch (1999: 3) bemerkt, dass zwischen Sprache und Kultur die dreierlei verschiedenen Relationen vorkommen. Die Sprache drückt aus und bestimmt nicht nur die kulturelle Realität einer Gesellschaft. Sie ist auch ein Werkzeug, mit dessen Hilfe die Menschen ihre Einstellung zu einer Sache oder zu einem Problem beschreiben und miteinander kommunizieren können. Außerdem bestimmt und symbolisiert sie eine kulturelle Realität, in der sie als ihr Bestandteil funktioniert. Wenn ein Mitglied einer Gesellschaft seinen Kommunikationspartner eine Nachricht überweisen möchte, hat er viele Möglichkeiten dazu. Man kann eine Nachricht per Telefon. Brief. E-Mail oder SMS übermitteln. Man kann sich treffen oder seine Botschaft in der Zeitung, in der Zeitschrift oder im Internet drucken. Bei der betreffenden Vermittlungsform der Information können verschiedene Sprachmittel verwendet werden. Sie werden alle durch einen inneren (d.h. von der Kultur abhängigen) Wirklichkeitsbegriff einer Gemeinschaft repräsentiert. Man muss bemerken, dass die kulturelle Wirklichkeit ein synchronischer Begriff ist und sich auf den Momentan-Zustand einer Gesellschaft bezieht. Die Wahlmöglichkeiten zur Informationsvermittlung innerhalb einer Kommunikationsgemeinschaft sind von ihrem jeweiligen Entwicklungsstand abhängig. Heutzutage ist das Nutzen des Handys oder des Computers so offensichtlich, dass niemand darüber nachdenkt, was er machen würde, wenn das kulturelle Entwicklungsniveau der Gesellschaft diese Art und Weise der Informationsvermittlung nicht bieten würde. Jede Sprache besitzt auch ein bestimmtes Zeichensystem. Diese in der bestimmten kulturellen Wirklichkeit existierenden sprachlichen Zeichen machen eine Realitätsbeschreibung aus. 
Das ist aber nur eine symbolische Beschreibung. Wenn zum Beispiel die Mitglieder der polnischen Gesellschaft sagen wollen, dass sie ein Objekt sehen, das vier Räder hat und sich selbständig bewegt, benutzen sie das Wort „samochód“. Dieses Wort symbolisiert ein Objekt der kulturellen Wirklichkeit und jedes Mitglied der Gesellschaft wird wissen, worum es geht.

\section{Der Begriff des "Interkulturellen Lernens und Lehrens"}

Viele Autoren und Sprachwissenschaftler (u.a. Doyé 1994: 43; Gnutzmann 1994: 65; Krumm 1994: 116) beklagen die Ungenauigkeit des Begriffs Interkulturelles Lernen (IKL). Dies verwundert nicht, weil im Zusammengang mit diesem Begriff solche Begriffe wie z.B. interkulturelle Kommunikation, interkulturelle Kommunikationsfähigkeit sowie Fremdverstehen genannt werden. Der Begriff des interkulturellen Lernens könnte nach Solmecke (1994: 165) der älteren Landeskunde einfach ersetzen und folglich keine Unterscheidung mehr getroffen wird. Etwas präziser bedeutet dies, dass der Lernende in einem prinzipiell unabschließbaren Lernprozess bestimmte Kenntnisse aus dem Bereich der eigenund fremdkulturellen Normen, Werte und Verhaltensmuster erwirbt. Der Besitz solcher Kenntnisse und solchen Wissens ermöglicht den Lernenden, sich innerhalb und gegenüber einer Zielsprachenkultur offen zu verhalten und dabei fähig und bereit ist, vor diesem Hintergrund seine eigene Kultur kritisch zu reflektieren (vgl. Leupold 2007: 277). Die Idee dieses Lernprozesses besteht darin, dass der Schüler lernen soll, Verschiedenheit zwischen Menschen und Kulturen $\mathrm{zu}$ tolerieren, die Angehörigen anderer Kulturkreisen sensibel zu betrachten und durch die Entdeckung und das Erlernen des Fremden die eigene Kultur aus einer anderen, neuen Perspektive wahrzunehmen und zu behandeln.

Zusammenfassend lässt sich feststellen, dass das interkulturelle Lernen die Perspektive einer Wechselbezüglichkeit von eigener und fremder Kultur eröffnet. Die Hauptrolle spielt hier die Interdependenz von Kulturen, nicht die Entdeckung der Kulturen separat ab.

\section{Interkulturelles Lernen und Lehren im Fremdsprachenunterricht}

Der Begriff des interkulturellen Lernens "hielt lange Zeit im Hintergrund". Es gab keine Fachdiskussion, die mit diesem Thema verbinden würde. Erst seit Mitte 
der 70er Jahre kann man einige Notizen darüber finden². Interkulturelles Lernen wurde dann als die Korrelation und Integration von Sprache, Sprachunterricht und Kulturkunde verstanden (Adamczak-Krysztofowicz 2003: 34f.). Die Theorie von einem Zusammenhang zwischen Sprache und Kultur wird in den 90er Jahren von vielen anderen Forschern und Wissenschaftlern aufgenommen ${ }^{3}$. Viele von ihnen unternehmen den Versuch, den Begriff des interkulturellen Lernens zum Schlüsselbegriff des Fremdsprachenlernens zu machen (Buttjes 1991: 2) und ihn als ein Ziel der Fremdsprachendidaktik zu bestimmen (Adamczak-Krysztofowicz 2003: 35).

Die Kultusministerkonferenz von 1996 hat in ihren Richtlinien viel Kraft dem Thema der Interkulturalität in der Schule gewidmet und den Bildungsauftrag postuliert, dass alle Menschen gleichwertig und ihre Wert- und Normenvorstellungen und ihre kulturellen Orientierungen achten sein sollen (SchmidtBernhardt/Stork/Adamczak-Krysztofowicz/ Rybszleger 2011: 87). Schnabel und Bianchi Schaeffer (2008: 8) betonen, dass „Schule bei der interkulturellen Bildung und Erziehung von Kindern und Jugendlichen Einstellungen und Verhaltensweisen fördern sollte, die dem ethischen Grundsatz der Humanität und den Prinzipien von Freiheit und Verantwortung, von Solidarität und Völkerverständigung, von Demokratie und Toleranz verpflichtet sind.“ Dementsprechend besteht die Aufgabe der Schule darin, dazu zu führen, kulturelle Verschiedenheit als wertvolle Sammlung und begehrenswerte Aufforderung zu erfahren (Schmidt-Bernhardt/Stork/Adamczak-Krysztofowicz/ Rybszleger 2011: 87).

Das Konzept des interkulturellen Lernens wird von vielen Fremdsprachendidaktikern unterschiedlich verstanden und sie nehmen verschiedene Stellung zur Sache, wenn es sich um den Entwurf handelt. Hunfeld (1994: 94) betont, dass Fremdsprachenlernen schon immer interkulturell ist. Nach Krumm (1994: 116ff.) sind interkulturelle Inhalte im Fremdsprachenunterricht nicht neu. Des Weiteren führt er aus, dass die Wahrnehmung durch die kultur-kontrastive Handlungen trainiert wird. Solmecke (1994: 165) ist aber der Meinung, dass man den Begriff durch die ältere Landeskunde ersetzen.

Interkulturelles Lernen und interkulturelle Kommunikation sind Begriffe, die als Zielvorstellungen der gegenwärtigen Fremdsprachendidaktik und des modernen Fremdsprachenunterrichts wahrgenommen werden (Kaikkonen 1997: 78). Die Voraussetzungen und Ideen interkulturellen Lernens sprechen dafür aus,

\footnotetext{
2 Mehr dazu Vereščagin/Kostomarov (1972), Pfeiffer $(1977,1980)$.

3 Dazu gehören z.B.: Krumm (1994), Solmecke (1994), Zawadzka (1997), Szczodrowski (1997), Pfeiffer (2002).
} 
„dass sich die Begegnungen und Kommunikation mit anderen Menschen ohne beiderseitiges Lernen reibungslos oder erfolgreich nicht abspielen können“ (ebd.). Interkulturelles Lernen wird heutzutage als ein relevantes Ziel der schulischen Erziehungstätigkeit betrachtet und der heutige Fremdsprachenunterricht zielt besonders auf interkulturelles Lernen (Kaikkonen 2002: 3). Alle können sich in der heutigen Schule den Fremdsprachenunterricht ohne Einführung der interkulturellen Inhalte oder ohne Entwicklung der interkulturellen Kompetenz.

Jedes Individuum, jeder Mensch kommt zur Welt in einem bestimmten Kulturkreis. Diese Kulturumgebung schenkt ihm ihre Sprache, ein System von Normen und Werten, Kommunikations- und Verhaltensmuster und -modelle usw. Kenntnis in eigener Kultur spielt eine sehr relevante Rolle, weil Fremdes immer aus der eigenen Perspektive betrachtet wird (ebd.). Interkultureller Fremdsprachenunterricht bringt solche Begriffe wie Rassismus, Ethnozentrismus, Xenophobie, Nationalismus, Diskriminierung näher. Dadurch lehrt man auch tolerant $\mathrm{zu}$ sein. Interkulturelles Lernen schenkt Aufmerksamkeit der interkulturellen Zusammenarbeit, der europäischen Integrationsentwicklung. Eine bedeutende Rolle in diesem Prozess spielt die Öffnung der Grenzen zwischen Ost und West. Interkulturelles Lernen macht uns auch aufmerksam auf die weltweiten Probleme, wie z.B. Umweltfrage, Überbevölkerung, Kriege, Ungleichgewicht in der Verteilung der Ressourcen.

\section{Die Elemente des interkulturellen Lernens und Lehrens im DaF-Unterricht}

Interkulturelles Lernen und Lehren stehen in enger Verbindung mit dem Begriff der Authentizität. Diese Konzepte verbinden mit persönlichen Erfahrungen und die Bedeutung von Lehrinhalten (Kaikkonen 2002: 11). Nach Pauli Kaikkonen (ebd.) spielen die authentischen Texte und Kontakte mit Vertreten der fremden Kultur, dank ihrer reichhaltigen Auswahl an authentischen Elementen, eine sehr relevante Rolle. Die Einführung authentischer Materialien sollte ein fester Bestandteil des Fremdsprachenunterrichts sein. Es scheint, dass die Suche nach einem perfekten authentischen Text für einen Fremdsprachenunterricht für den Lehrer nicht allzu kompliziert ist, insbesondere im Zeitalter des Zugangs zum Internet und des einfachen Zugangs zu Informationen. Leider kommt es häufig vor, dass der ausgewählte Text die Erwartungen der Schüler nicht erfüllt und ihnen viele Schwierigkeiten bereitet. Erstens sind authentische Texte für Lernende $\mathrm{zu}$ linguistisch schwierig, d. H. Sie enthalten ein unbekanntes 
Vokabular, oder "gespickt" sind schwierige grammatikalische Strukturen. Das Problem können auch Analogien zu historischen Ereignissen oder zur Kultur des Sprachraums sein, die für sie aufgrund unzureichender Kenntnisse der Schüler unleserlich sein können. Eine weitere Schwierigkeit ist das Volumen des Textes. Sie sind oft $\mathrm{zu}$ lang und motivieren daher nicht, mit ihnen $\mathrm{zu}$ arbeiten. Trotz ungewöhnlicher Probleme bei der Wahl des Textes für den Fremdsprachenunterricht sind sie jedoch ein besonderer Wert als echtes Objekt fremder Kultur. Sie dienen oft auch als visuelle Informationsquelle. Betrachten wir Fotos oder Illustrationen in einer farbigen Presse, lernen wir viel über die Kultur einer bestimmten Gemeinde, beispielsweise lernen wir die Art der Kleidung, die Wohnbedingungen oder Verhaltensweisen von Vertretern anderer Kulturen kennen. Fernsehprogramme oder Radioprogramme stellen jedoch reale Kommunikationssituationen dar, in denen wir als Empfänger verbale und nonverbale Kommunikationsmethoden erleben. Neben authentischen Materialien spielen authentische Treffen mit Vertretern anderer Kulturen eine sehr wichtige Rolle beim Unterrichten einer Fremdsprache. Krystyna Mihułka (2013: 60) definiert als interkulturelles Treffen ,jeden Kontakt mit einer Fremdsprache und der darin reflektierten Kultur (...), in der unsere eigene, uns vertraute und uns vertraute mit dem Neuen, Anderen, Fremden zusammentreffen“. Das Aufeinandertreffen mit neuer, fremder Sprache und damit verbunden einer anderen Kultur führt zu Vergleichen neuer Kuriositäten, kultureller Einzigartigkeit mit Erfahrungen, die mit der Kommunikation mit der einheimischen Kultur verbunden sind. Diese Treffen können extreme Emotionen von Euphorie bis Frustration hervorrufen, was zur Bildung von Stereotypen und Vorurteilen gegenüber anderen Nationalitäten, mangelndem Selbstvertrauen, Kulturschock und Abneigung gegen alles Neue und Fremde führt. Diese Kontakte können aber auch zu einem erwartungsgemäßen positiven Ende führen und die Entwicklung von Kompetenzen von sprachlich über kommunikativ bis interkulturell anregen (ebd.). Durch diese Kontakte erhalten die Studierenden die Möglichkeit, eine ausreichende Kompetenz in kommunikativen interkulturellen Situationen zu erlangen, was Rogers/Steinfatt (1999: 222) jedoch nicht garantiert. Die Qualität dieser Treffen spielt eine entscheidende Rolle. Wie von Apeltauer (1994: 3ff.) betont, hängt die Qualität dieses Treffens zwischen Vertretern verschiedener Kulturen und Gemeinschaften in hohem Maße von den Kenntnissen ab, die sich auf den historischen Hintergrund des Gesprächspartners sowie auf situative und individuelle Faktoren beziehen. Einer der Hauptvorteile interkultureller Begegnungen ist die Möglichkeit längerer persönlicher Kontakte zu Vertretern der fremden Kultur. Persönliche Treffen - im Idealfall auch Freundschaft und Anerkennung 
der Identität anderer - bringen den Parteien gegenseitigen Nutzen. Vor allem motivieren sie die Schüler, die Sprache und Kultur eines Freundes, eines Kollegen, zu lernen. Persönliche oder postalische Kontakte werden auch zum Informationsaustausch verwendet.

Trotz der verschiedenen Meinungen über das interkulturelle Lernen und Lehren kann man feststellen, dass das Verdienst des interkulturellen Ansatzes im fremdsprachlichen Unterricht unschätzbar ist und beruht nach Roche (2001) darauf, die Korrelation zwischen sprachlichem und kulturellem Lernen wieder stärker zu präsentieren. Schmidt-Bernhardt/Stork/Adamczak-Krysztofowicz/ Rybszleger (2011: 87) betonen, dass das Hauptziel des interkulturellen Lernens in der Schule und in Studium besteht darin, die Lernenden zu einem angemessenen Handeln fähig zu machen und sie für „ein respektvolles und akzeptierendes Zusammenleben verschiedener Kulturen zu sensibilisieren“.

\section{Interkulturelles Lernen als offizielles Postulat für den modernen Fremdsprachenunterricht}

Die didaktischen Konzeptionen wiederspiegeln die aktuellen sozialzivilisatorischen Bedingungen und Denktendenzen (vgl. Wilczyńska 2010: 113). Kein Wunder, dass sich der moderne Fremdsprachenunterricht auf interkulturelles Lernen bezieht (Kaikkonen 2002: 3). Diese Meinungsund wissensbildende Prozesse im Bereich der Didaktik können gebildet werden und sie sind durch die aktive Bildungspolitik auf Niveau der Europäischen Union und der bestimmten Mitgliedsländer geprägt (vgl. Wilczyńska 2010: 113). Man nimmt derzeit an, dass strikt sprachliche Kompetenzen (Wissen der Phonologie, der Morphologie, der Lexik, der Semantik und der Syntax) nicht ausreichend sind, damit der Lernende den kommunikativen Erfolg erreichen kann $^{4}$ (vgl. Chłopek 2009: 61). Die gegenwärtigen Modelle der Kommunikationskompetenz berücksichtigen das (inter)kulturelle Wissen und Bewusstsein, die eine Bedingung sind, an dem sehr wichtigen für heutigen Europa interkulturellen Dialog erfolgreich teilzunehmen (ebd.).

Schon am Anfang des europäischen Integrationsprozesses, nach den Grausamkeiten des zweiten Weltkrieges, haben die Väter der ersten Europäischen Gemeinschaften - Jean Monnet und Robert Schuman einen visionären und gleichzeitig realistischen Bauplan von Europa. Er hat auf die Zusammenarbeit, nicht auf die militär-politische Rivalität beruht (vgl. Wilczyńska 2010: 114).

${ }^{4}$ Mehr dazu Bachman (1990), Europarat (2001/2003). 
Der seit 1971 aufgenommene Gemeinsame europäische Referenzrahmen für Sprachen: lernen, lehren, beurteilen, in Polen gekannt als Europejski system opisu kształcenia językowego: uczenie się, nauczanie, ocenianie (ESOKJ)5, der seit September 2000 entscheidend (englischsprachigen) Version ${ }^{6}$ publiziert ist, kann als Versuch verstanden werden, eine gemeinsame Basis für die Entwicklung von zielsprachlichen Lehrwerken, Prüfungen, Lehrplänen und Richtlinien usw. für ganz Europa zu bilden. Er definiert Kompetenzniveaus, die dem Lernenden ermöglichen, Vorschritte auf jeder Stufe des lebenslangen Lernens von Fremdsprachen zu messen und erleichtert den Vergleich und Anerkennung von Sprachqualifikationen. Dieses Dokument trägt auch zur Stärkung der Zusammenarbeit in Europa im Sprachenlernen bei und entwickelt die Mehrsprachigkeit und Kulturbewusstsein der Gesellschaft. Seine Rolle besteht auch darin, zur Reflexion und Diskussion über das Sprachenlernen- und Lehren anzuregen und die Mobilität in Europa zu unterstützen.

Die fundamentale Rolle interkulturellen Lernens hat auch in den offiziellen Dokumenten einen Ausdruck gefunden. Dieser Aspekt wird als unbedingt erforderlicher Bestandteil des modernen und zukunftsorientierten Fremdsprachenunterrichts angesehen. Der Beirat „Deutsch als Fremdsprache“ des Goethe-Instituts hat 1997 ein Thesenpapier formuliert, dem eine wegweisende Funktion beim Lernen und Lehren von Deutsch als Fremdsprache zugeschrieben werden kann.

Man muss auch betonen, dass die Politik der Europäischen Kommission, die als Ziel den Schutz und die Vermarktung der sprachlich-kulturellen Verschiedenheit Europas hat, übereinstimmend mit Aufzeichnungen der wesentlichen internationalen Urkunden ist. Dazu gehört zum Beispiel Konwencja dotycząca ochrony i promocji różnorodności ekspresji kulturalnych (dt. Die des Schutzes und der Vermarktung betreffende Konvention der Verschiedenheit der kulturellen Expressionen), die von UNESCO (2005) bearbeitet wurde. Sie wurde im Jahr 2009 von Polen ratifiziert. Sie definiert Interkulturalität als gleiche Interaktionen zwischen verschiedenen Kulturen und Produkten der kulturellen Expressionen, die im Dialog und in der gegenseitigen Achtung mitgeteilt werden (Wilczyńska 2010: 114).

Der im September 2008 von Europäischer Kommission publizierte Bericht hält und aktualisiert die schon in Lissabon festgelegte Strategie im Bereich der Entwicklung der Mehrsprachigkeit. Diese Strategie beinhaltet viele

\footnotetext{
${ }^{5}$ Seit 2003 funktioniert eine polnische Version.

${ }^{6}$ Seit Februar 2001 existiert auch eine deutsche Version, abrufbar auf der Homepage des GoetheInstituts (http://www.goethe.de/z/50/i0/htm).
} 
koordinierten Handlungen auf verschiedenen Ebenen - auf lokalem, regionalem, inländischem und Unionsebene. Dazu gehören u.a.:

- der Druck auf die weitere Verallgemeinerung der Lissabonner Weisung, die die allgemeine Kenntnisse zwei anderen Fremdsprachen neben der Muttersprache betrifft, auch in Bezug auf Erwachsene, die keinen Anlass zum Lernen anderer Sprache hatten, oder sie dieses Lernen aufgegeben haben,

- die Einführung des Fremdsprachenlehrens in dem ganzen Bildungssystem, auch in der Berufsbildung,

- die weitere Vermarktung der Mehrsprachigkeit, und die bessere Ausnutzung schon existierenden Potenzial durch den Austausch der guten Praktiken im Bereich des zwischenkulturellen Dialogs,

- die Ausarbeitung der Partnerschaft im Bereich der Entwicklung der Mehrsprachigkeit und des interkulturellen Dialogs auch mit den Ländern der Dritten Welt, also aus der Europäischen Union (http://ec.europa.eu/education/languages/pdf/com/2008_0566_pl.pdf).

Wilczyńska (2010: 122) betont, dass man die besondere Verbesserung der Situation durch zwei folgende Handlungen erreichen könnte:

- die Unterstützung der Herstellung von Filmen und Programmen in origineller Version mit Untertiteln und die Verallgemeinerung der europäischen medialen Produktionen,

- Projekte, die die Entwicklung und Verallgemeinerung der sprachlichen und kommunikativen Technologien fordern.

Obwohl sich fast keinen Lehrer vorstellen kann, den Fremdsprachenunterricht ohne Einführung der interkulturellen Inhalte zu führen, gibt es in der modernen Bildungssystem kleine Mängel. Chłopek (2009: 67) nach der Durchführung der Fragebogenuntersuchung betreffend des Kulturlehrens im Fremdsprachenunterricht in Polen hat bestimmte Forderungen für die Zukunft gegeben:

- mehr Stunden der Fremdsprachen,

- bessere Lehrwerke. Die heutigen Lehrwerke entwickelt vor allem die strickte sprachlichen Kompetenzen. Die neuen Lehrwerke sollten auch die interkulturellen Aspekte lehren. Diese Inhalte sollten auch ein integraler Teil der in dem Lehrwerk enthaltenen Texten und Übungen sein und den Lernenden zur Reflexion und Diskussion anregen,

- das bessere Angebot der ergänzenden Materialien auf dem Markt, 
- Kurse und Schulungen für die (zukünftigen) Lehrer, die fundierte Kulturinformationen und auch die methodische Vorbereitung zur Vermittlung dieser Inhalte anbieten sollten,

- die Reflexion der Lehrer auf die Art der entwickelten Kompetenzen durch die von ihnen angewendeten Lehrwerken und Materialien,

- die Untersuchung des Bewusstseins der Lernenden. Sie sollten wissen, dass nicht nur strikt sprachliches Wissen die ungestörte Kommunikation garantiert. Die Lernenden müssen sich dessen bewusst sein, dass Mangel an Kenntnis der kulturellen Unterschiede ein Grund vieler Missverständnisse sein kann.

Neben diesen Postulaten gibt es auch zahlreiche andere - alle von ihnen sind relevant und alle sollten ins Leben gerufen werden. Der Lernende, der sich über die kulturellen Unterschiede und über die Existenz des kulturellen Bestandteils im Laufe der Kommunikation im Klaren ist, wird bessere Motivation zum Lernen der Fremdsprache haben als der Lernende ohne dieses Wissen. Er wird auch größere Sensibilität und Toleranz für die Vertreter anderer Kulturen haben. Aus diesem Grund wird die Fremdsprache ein Werkzeug für die Erkenntnis anderer Kulturen und Nationen.

\section{Bibliografie}

Adamczak-Krysztofowicz, S. (2003). Texte als Grundlage der Kommunikation zwischen Kulturen. Eine Studie zur Kultur- und Landeskundevermittlung im DaF-Studium in Polen. Verlag Dr. Kovac: Hamburg.

Aleksandrowicz-Pędlich, L. (2005). Międzykulturowość na lekcjach języków obcych. Białystok: Wydawnictwo Uniwersytetu w Białymstoku.

Andrzejewski, B. (2005) Poznanie i komunikacja. Koszalin: Wydawnictwo Uczelniane Politechniki Koszalińskiej.

Apeltauer, E. (1994). „Einleitung“. [w:] Apeltauer, A. (red.): Aus Erfahrung lernen: Exkursionen und Auslandspraktika im Bereich Deutsch als Zweitund Fremdsprache, Hohengehren: Schneider Verlag (=Interkulturelle Erziehung in Praxis und Theorie, t. 16), s. 1-16.

Banach, B. (2003). ,Język a kultura”. [w:] Języki Obce w Szkole 2/2003, s. 3-5.

Brislin, R. W. (1981). Cross - cultural Encounters. New York: Pergamon Press.

Burwitz-Melzer, Eva (Hrsg.) (2003). Allmähliche Annäherungen: Fiktionale Texte im interkulturellen Fremdsprachenunterricht der Sekundarstufe I. Tübingen: Narr.

Buttjes, D. (1991). "Interkulturelles Lernen im Englischunterricht". [w:] Der fremdsprachige Unterricht Englisch, 1/1991, s. 2-8.

Chłopek, Z. (2009). "Nauczanie kultury na lekcjach języka obcego w Polsce: Wyniki badań kwestionariuszowych". [w:] Języki Obce w Szkole, 1/2009, s. 61-68. 
Grzybowski, P. P. (2011). Edukacja międzykulturowa - konteksty. Od tożsamości po język narodowy. Oficyna Wydawnicza "Impuls".

Hunfeld, H. (1994). "Fern vom "versöhnten Zustand". Anmerkungen zum interkulturellen Lernen im FU". [w:] Bausch, K.-R. / Christ, H. / Krumm, H.-J.: Interkulturelles Lernen im FU. Arbeitspapiere der 14. Frühjahrskonferenz zur Erforschung des FUs. Tübingen: Narr, s. 94-100.

Jańska, M. U. (2006). Interkulturelles Lernen in der bilingualen deutsch-polnischen Erziehung. Evaluation der Unterrichtsmaterialien für den frühen fremdsprachlichen Deutschunterricht in Polen. Wrocław/ Dresden: Oficyna Wydawnicza ATUT/ Neisse Verlag.

Kaikkonen, P. (1997). „Fremdverstehen durch schulischen Fremdsprachenunterricht“. [w:] INFO DaF24, 1, s. 78-86.

Kaikkonen, P. (2002). „Authentizität und authentische Erfahrung in einem interkulturellen Fremdsprachenunterricht“. [w:] Info DaF 29, 1, s. 3-12.

Koniewicz, D. M. (1997). „Aspects of Cultural Relativity in EFL Handbooks”. [w:] Lewandowska-Tomaszczyk, B. (ed.), New Trends in Language Studies. Łódź: Wydawnictwo Uniwersytetu Łódzkiego. Folia Linguistica 36.

Kramsch, C. (1999). Language and culture. Oxford: CUP.

Krumm, H.-J. (1994). „Interkulturelles Lernen im FU“. [w:] Bausch, K.-R. / Christ, H. / Krumm, H.-J.: Interkulturelles Lernen im FU. Arbeitspapiere der 14. Frühjahrskonferenz zur Erforschung des FUs. Tübingen: Narr, s. 116-127.

Leoński, J. (2005). „Wielokulturowość: dylematy wyborów światopoglądowych“. [w:] Oświata $i$ Wychowanie 4/2005, s. 12-13.

Leupold, E. (2007). Französisch unterrichten. Grundlagen- Methoden- Anregungen. 4. Aufl. Seelze-Velber: Klett-Kallmeyer.

Malinowski, B. (1923). „The Problem of Meaning in Primitive Language”, [w:] Ogden, Ch., Richards, J. A. The Meaning of Meaning, London: Kegan, Paul, Trench, Trubner and Co.

Mihułka, K. (2008). „Dialog interkulturowy jako piąta sprawność na lekcji języka obcego”. [w:] Języki Obce w Szkole, 2/2008, s. 13-19.

Mihułka, K. (2010). Stereotype und Vorurteile in der deutsch-polnischen Wahrnehmung: eine empirische Studie zur Evaluation des Landesbildes durch Germanistikstudenten. Rzeszów: Wydawnictwo Uniwersytetu Rzeszowskiego.

Mihułka, K. (2013). „Spotkania interkulturowe z perspektywy uczenia się i nauczania języka obcego". [w:] Języki Obce w Szkole, 02/2013, s. 60-69.

Odelski, M. R. (2005). „Komunikacja interkulturowa jako przykład pomostu między kulturami“. [w:] Neofilolog, 27/2005, s. 21-24.

Pawłowska, A. (2010). „Rozwijanie kompetencji interkulturowej na lekcji języka obcego”. [w:] Języki Obce w Szkole, Nr 1/2010, Warszawa, s. 58-66.

Pfeiffer, W. (2001). Nauka języków obcych. Od praktyki do praktyki, Poznań: Wagros.

Rogers, E. M./ Steinfatt, T. M. (1999). Intercultural Communication, Waveland Press, Incorporated. 
Schmidt-Bernhardt, J./ Stork, A./ Adamczak-Krysztofowicz, S./ Rybszleger, P. (2011). Deutsch - polnische Entdeckungen. Projektarbeit zur Förderung interkultureller Kompetenz in Schule und Studium. Theoretische Grundlagen und praktische Lernmaterialien, Marburger Schriften zur Lehrerbildung, Band 6, Marburg: Tectum Verlag.

Schmölzer-Eibinger, S./ Weidacher, G. (eds.) (2007). Textkompetenz. Eine Schlüsselkompetenz und ihre Vermittlung. Tübingen: Narr.

Schnabel, B./ Bianchi Schaeffer M. (Hrsg.) (2008). Das interkulturelle Klassenzimmer. Potentiale entdecken. Anregungen für Lehrerinnen und Lehrer. Frankfurt/Main: Brandes und Apsel.

Solmecke, G. (1994). „Interkulturelles Lernen im FU“. [w:] Bausch, K.-R. / Christ, H. / Krumm, H.-J.: Interkulturelles Lernen im FU. Arbeitspapiere der 14. Frühjahrskonferenz zur Erforschung des FUs. Tübingen: Narr, s. 165-171.

Szopski, M. (2005). Komunikowanie międzykulturowe. Warszawa: WSiP.

Widawska, B. (2006). „Kształcenie rodzimej kompetencji kulturowej a komunikacja (inter)kulturowa“. [w:] Języki Obce w Szkole, 6/2006, s. 19-23.

Wilczyńska, W. (2010). „Przygotowanie do komunikacji interkulturowej - wytyczne polityki europejskiej a dydaktyka języków obcych”. [w:] Mackiewicz, Maciej: Kompetencja interkulturowa $w$ teorii $i$ praktyce edukacyjnej, Poznań: Wydawnictwo Wyższej Szkoły Bankowej w Poznaniu, s. 113-123. 